\title{
HIGH ASPECT RATIO SILICON MICROSTRUCTURES FOR NUCLEIC ACID EXTRACTION
}

\author{
Lee A. Christel, Kurt Petersen, William McMillan, Gregory T. A. Kovacs \\ Cepheid \\ 1190 Borregas Ave \\ Sunnyvale, CA 94089
}

\begin{abstract}
Silicon fluidic microchips with high surface area to volume ratios have been fabricated using deep reactive ion etching (DRIE) technology $[\mathbf{1 , 2}]$. These microchips have been used as capture surfaces for nucleic acids (NA). Short (500 bp) and medium size (48,000 bp) DNA have been captured, washed, and eluted with these chips using glass binding chemistries. DNA quantities approaching $40 \mathrm{ng} / \mathrm{cm}^{2}$ of binding area were captured from input solutions in the $100-1000 \mathrm{ng} / \mathrm{mL}$ concentration range, and detected using fluorescence techniques. For low concentration studies, PCR and gel electrophoresis were used as the detection method. Extraction efficiencies of about $50 \%$, and concentration factors of about 10 have been demonstrated using bacteriophage lambda DNA as the target. From an input volume of $500 \mu \mathrm{L}$ containing $5 \times 10^{4}$ DNA molecules, $2-3 \times 10^{4}$ DNA molecules were extracted and eluted into a $25 \mu \mathrm{L}$ volume. These results demonstrate the viability of utilizing such microchips as an element in a compact, disposable cartridge format for the detection of DNA in applications such as clinical diagnostics, biowarfare agent detection, food quality control, and environmental monitoring.
\end{abstract}

\section{INTRODUCTION}

Important diagnostic tests such as HIV viral load in the blood of clinical AIDS patients depend on the detection of small quantities of DNA or RNA. Such tests utilize polymerase chain reaction (PCR) [3] to amplify (multiply) the number of copies of a target sequence to a detectable level. The PCR technique requires a relatively pure DNA sample in aqueous solution, free of contaminants that inhibit the PCR process. Such inhibitors include heavy metals, such as the iron in hemoglobin, nucleases, certain proteins and enzymes found in raw biological samples. There is thus a need for methods to extract and purify DNA from a variety of samples.

Many DNA purification kits are available commercially [4], each for a particular DNA or RNA and input sample. Typically, these kits utilize some form of silica gel, glass matrix, or membrane as the capture medium since it is known that NA will bind to glass or other silica-type surfaces under the proper chemical conditions [5]. A typical protocol for DNA purification involves a number a steps whereby various reagents are added to the sample and the sample is centrifuged to separate precipitated components from the solution. Such a procedure is quite involved, and in many cases is still done manually. Although such a procedure can be automated using robotics, integrating such a protocol into a low cost, disposable analysis format requires a reexamination of the entire process.

As the broad application of the PCR technique continues to be refined and recognized, there is enormous market pressure to improve the cost and throughput of DNA/RNA tests. There are many applications where a small DNA analysis tool utilizing disposable cartridges would expand markets if the costs and throughputs can be improved. Such cartridges would likely combine low-cost plastics technology with selected micromachined components for critical functions such as DNA extraction. The motivation for this work is the broader goal of developing an automated analysis system which takes raw sample inputs and gives a PCR result in a few minutes. Flow-through cartridge designs capable of reagent containment, mixing and delivery for the processing of significant clinical sample volumes, while maintaining small internal volumes of critical components, appear promising. DNA extraction chips are an important component of this strategy.

We have fabricated high surface area DNA capture surfaces of oxidized silicon using deep reactive ion etching (DRIE) and are investigating the ability of these structures to controllably capture and release DNA from test solutions. Several parameters are of primary importance if such a chip is to be commercially viable. The chip must have sufficient binding capacity to retain the quantity of DNA required for the subsequent assay. It must extract the DNA efficiently, i.e. the ratio of target DNA captured to target DNA input must be high (exceed $50 \%$ ). It must be able to complete the extraction in a reasonable amount of time, i.e. the capture must occur at reasonable flow rates, so that clinically realistic volumes (sometimes several $\mathrm{mL}$ ) can be processed expeditiously. The chip must also concentrate the input DNA, allowing the DNA to be eluted (released) into a small volume for PCR amplification. These volumes are typically $100 \mu \mathrm{L}$ or less to conserve expensive PCR reagents. The chip must allow wash solutions to be efficiently passed through while retaining the DNA, so that contaminants, such as PCR inhibitors, can be excluded from the final elution without requiring large volumes of wash solution typically associated with commercial kits. Finally, the chip must have a reasonable cost, preferably less than one dollar, in order to be used in a disposable format. If the above conditions can be met, there is a large market opportunity for DNA assays in a wide range of markets, including clinical and veterinary diagnostics, food and environmental monitoring, forensics, and biowarfare detection.

\section{CHIP FABRICATION}

The process used to produce the DNA capture chips is shown in Fig. 1. This process is simpler than competing technologies such as LIGA [6]. Fabrication starts with $100 \mathrm{~mm}$ (100) silicon, double-side polished, approximately $400 \mu \mathrm{m}$ thick. The wafer is first oxidized to produce a thin pad oxide, and then coated with silicon nitride. The backside is patterned and etched in $\mathrm{KOH}$ to produce fluidic ports that are $200 \mu \mathrm{m}$ deep. The frontside is next patterned with the DRIE mask, and etched for approximately one hour in an STS DRIE plasma etcher to produce a field of up to 5,000 pillars, each being $200 \mu \mathrm{m}$ high. At the end of this etch, the frontside pattern meets the backside ports. The wafer is further oxidized to coat the internal surfaces with silicon dioxide. The silicon nitride prevents additional oxidation of the top surface. The nitride and underlying pad oxide are now removed by plasma and wet etching to produce a bare silicon upper surface. A cover of pyrex ${ }^{\mathrm{TM}}$ is anodically bonded to the wafer to finish the process. The wafer is then ready for sawing to produce the finished square die, which are $3.85 \mathrm{~mm}$ on a side. 

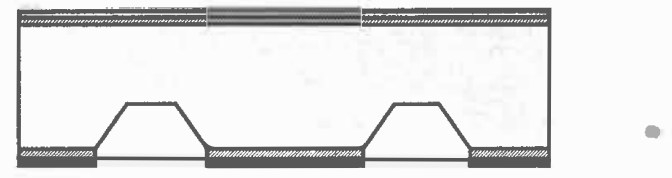

Deep RIE Etching:
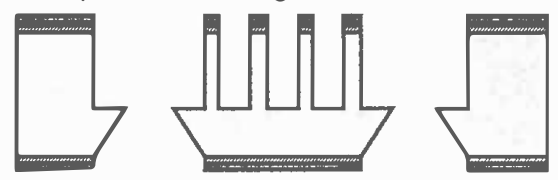

Reoxidation:

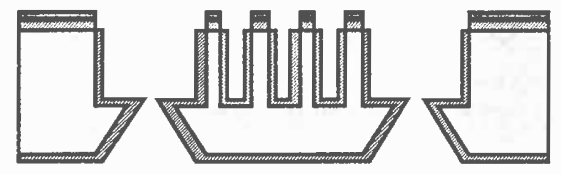

Nitride Strip and Anodic Bonding:
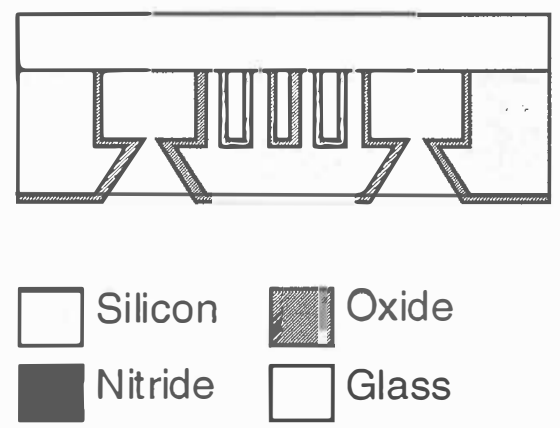

Figure 1. Process flow for the fabrication of high aspect ratio DNA capture surfaces in silicon

Figure 2 shows an SEM of one chip surface consisting of $200 \mu \mathrm{m}$ high columns, each about $20 \mu \mathrm{m}$ in diameter, with a pitch of $34 \mu \mathrm{m}$. Chips with pillar diameters of $10 \mu \mathrm{m}$ and a pitch of $18 \mu \mathrm{m}$ have also been made. After anodic bonding to the glass top cover, the chip has a total internal surface area of $36 \mathrm{~mm}^{2}$ in an 'active' area of $3.5 \mathrm{~mm}^{2}$.

\section{EXPERIMENTAL DETAILS}

The DNA capture studies reported here can be divided into two types: high concentration inputs (on the order of 100-1,000 $\mathrm{ng} / \mathrm{mL}$ ) and low concentration inputs (at or below $10^{5}$ copies of target DNA). These two regimes have relevance to different clinical situations. Samples involving genomic DNA, such as might be present in a sample containing lysed white blood cells, typically have large concentrations of DNA. On the other hand, samples used for diagnosis of infectious diseases often contain only very small amount of pathogenic DNA of interest. Serum, plasma, and urine are examples of the latter situation. For high concentration studies, the quantities of DNA are large enough that fluorescence techniques can be used to detect the DNA. For low concentrations however, PCR must be used to first amplify a target sequence, and only then can the presence of the target DNA be verified usually by gel electrophoresis. Quantitation of the PCR product is more difficult, and the use of calibration standards run through PCR in parallel is mandatory.

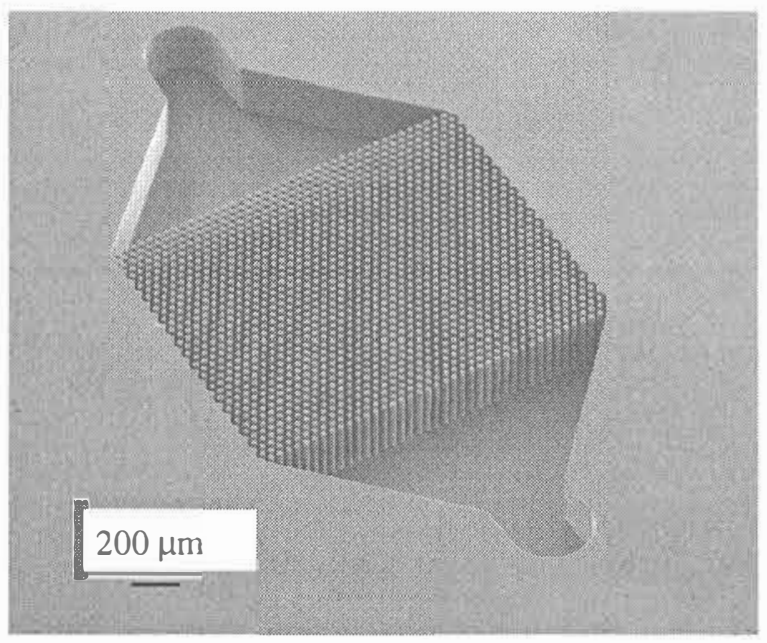

Figure 2. SEM of DNA Capture Chip Surface. These pillars are $200 \mu \mathrm{m}$ high with a pitch of $34 \mu \mathrm{m}$.

For DNA capture studies, a fluidic test system has been constructed. It consists of a chip holder, and several computer controlled syringe pumps and valves. A schematic representation of the fluidic system is shown in Fig. 3. An acrylic chip holder allows multiple fluidic connections to be made to the backside of the chip via 0.01 " ports and an elastomeric gasket between the chip and fixture. Two syringe pumps allow fluids to be aspirated from source reservoirs into storage loops, and dispensed either to the chip or to waste. Several distribution valves are used to control fluid routing. All valve and pump motions are controlled via the RS-232 interface of an IBM-compatible PC. Protocols are written and stored as stand-alone programs, insuring that the experimental conditions are consistent from run to run.

Starting solutions were obtained by diluting high concentration DNA stock solutions with a glass binding solution from a commercially available DNA purification kit [4]. This binding solution is a chaotropic salt solution, intended to both denature proteins that might be present in a real clinical sample, and to produce conditions that allow binding to glass. Such a solution is a good representation of the chemical environment that would be presented to the chip in a real clinical sample. Wash and elution reagents were also taken from the same commercial kit. The wash solution is typically an ethanol-based solution, with several other ionic components. Elution solutions are either TE (10 $\mathrm{mM}$ Tris- $\mathrm{HCl}$ and $1 \mathrm{mM}$ EDTA), or water.

A typical experimental protocol is as follows. The chaotropic DNA starting solution is first passed through the chip, during which DNA present in the solution binds to the silicon dioxide-coated microstructure. This is then followed by the ethanol-based wash solution. The wash solution is intended to wash away the salts and other PCR inhibitors that might be present in the sample. Finally an elution reagent is passed through the chip, releasing the DNA back into the fluid stream. This elution is collected in small aliquots for analysis.

For detection of elutions of high concentration DNA, a Carl Zeiss fluorescence microscope was used in conjunction with a photon-counting photomultiplier tube (PMT) and software from Photon Technologies, Inc (Monmouth Junction, NJ). The microscope was fitted with a filter set designed for fluorescein. This filter set allows excitation of the sample at $490 \mathrm{~nm}$, and detection at $515 \mathrm{~nm}$ and beyond. This setup is suitable for fluorescein-labeled DNA, as well as for several other DNA dyes, such as YOYO-1 and PicoGreen [7]. 


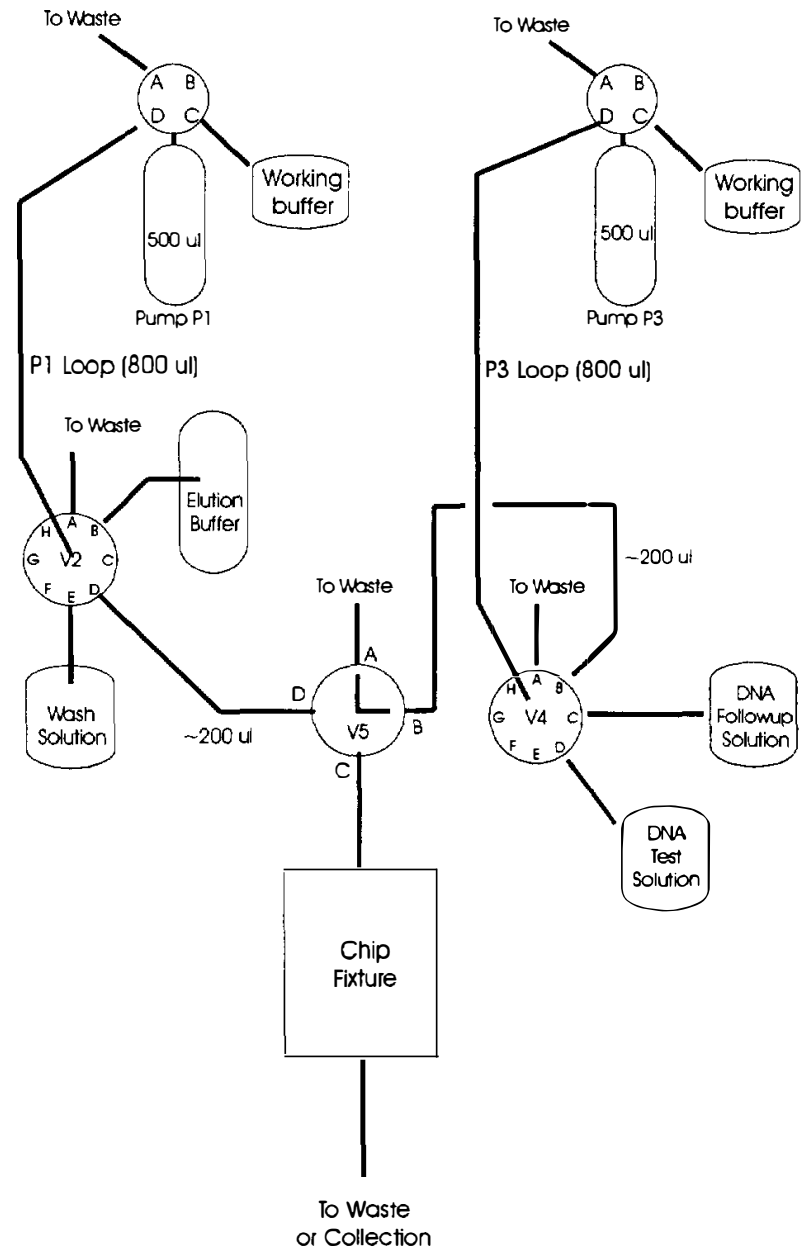

Figure 3. Schematic of Fluidic Test System.

For low concentration studies, chip output was processed through a standard PCR protocol using a Perkin Elmer 9700 thermal cycler. Reference samples spanning several orders of magnitude in target starting copy number were run in parallel. PCR product was then processed through gel electrophoresis, typically with $1 \%$ agarose gels and ethidium bromide staining. Photographs of the resulting gel bands were compared visually to the reference standards to estimate the starting copy number in the chip elutions. Work in progress is aimed at improving further the quantitation of the PCR product.

\section{HIGH CONCENTRATION RESULTS}

For high concentration studies, a solution of plasmid digest DNA from Bio-Rad was used as the starting material [8]. This solution consists of a mix of DNA with an average length of about $500 \mathrm{bp}$. The material is tagged with fluorescein, a fluorescent marker, allowing in-situ observation of the DNA during sample processing.

The chip elutions were collected into a Delrin fixture consisting of a number of small wells, each with a volume of approximately $25 \mu \mathrm{L}$. A number of $20 \mu \mathrm{L}$ elution droplets were captured into the wells. In parallel, a series of standards of known concentration were dispensed into separate wells. $5 \mu \mathrm{L}$ of $1.0 \mu \mathrm{M}$ YOYO-1 dye [7] were then added to each well. This dye further enhances the fluorescence of the samples. After a short incubation period, the wells were measured for signal using the fluorescence microscope/PMT system.

The above protocol was performed under several experimental conditions. The ranges of experimental parameters are given in Table 1. Note that the internal volume of the chip is about $0.2 \mu \mathrm{L}$, leading to a residence time of $200 \mathrm{msec}$ at a flow rate of $1.0 \mu \mathrm{L} / \mathrm{s}$.

Table 1. Experimental Conditions for High Concentration DNA Capture Experiments.

\begin{tabular}{||c|c||}
\hline \hline Parameter & Range \\
\hline DNA Conc. & $100-1000 \mathrm{ng} / \mathrm{mL}$ \\
\hline DNA Volume & $400 \mu \mathrm{L}$ \\
\hline DNA Total Dose & $40-400 \mathrm{ng}$ \\
\hline DNA Flow Rate & $0.1-5.0 \mu \mathrm{L} / \mathrm{s}$ \\
\hline Wash Volume & $400 \mu \mathrm{L}$ \\
\hline Wash Flow Rate & $0.5-5.0 \mu \mathrm{L} / \mathrm{s}$ \\
\hline Elution Flow Rate & $0.5 \mu \mathrm{L} / \mathrm{s}$ \\
\hline
\end{tabular}

The total binding capacity of high aspect ratio chips was investigated using an input of $400 \mathrm{ng}$ of DNA $(400 \mu \mathrm{L}$ of a 1,000 $\mathrm{ng} / \mathrm{mL}$ solution) followed by $400 \mu \mathrm{L}$ of wash and then elution. All flow rates were $0.5 \mu \mathrm{L} / \mathrm{s}$. The fluorescence signals from the sample wells, as well as those of the reference standards are shown in Fig. 4. By comparing each elution signal to the standards, and summing them, we conclude that 11-12 ng of DNA has been captured, then eluted from the chip. It has been reported [9] that the maximum binding capacity of glass is $\approx 40 \mathrm{ng} / \mathrm{cm}^{2}$. Since the internal surface area of the chip is $\approx 0.36 \mathrm{~cm}^{2}$, it is evident that in this experiment, an amount of DNA consistent with the maximum binding capacity of glass has been captured.

As a comparison, the same protocol was run utilizing a 'flat' chip without the enhanced surface area. This chip had an internal surface area of about $0.06 \mathrm{~cm}^{2}$. In this case, about $2.5 \mathrm{ng}$ of DNA was captured and eluted, very nearly $1 / 6$ of that captured on the DRIE chip. This is also consistent with binding at the capacity limit of glass.

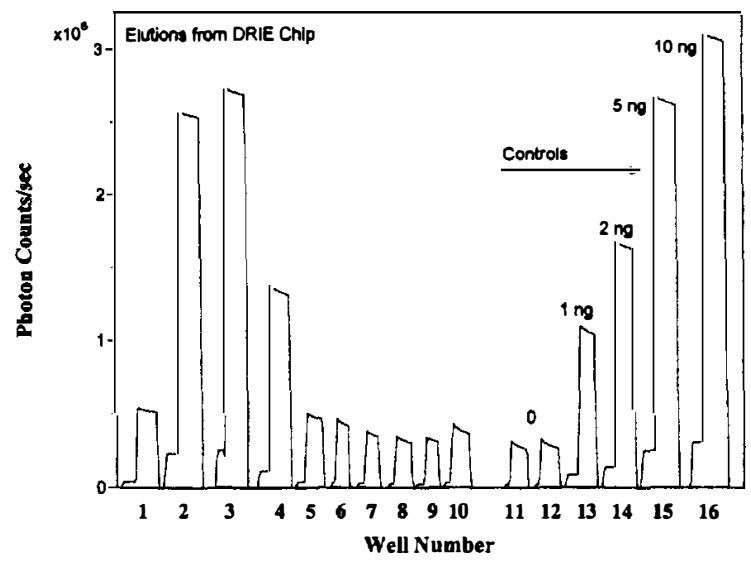

Figure. 4. Fluorescent signals from DNA chip elutions, with reference samples. The total DNA captured is about $12 \mathrm{ng}$.

Several other experimental conditions were examined. It was found that DNA flow rates higher than $0.5 \mu \mathrm{L} / \mathrm{s}$ led to reduced capture efficiency, but wash flow rates could be increased up to $5.0 \mu \mathrm{L} / \mathrm{s}$ without loss of efficiency. These results are summarized in Table 2. 
Table 2. Experimental Conditions for High Concentration DNA Capture Experiments. All input volumes were $400 \mu \mathrm{L}$.

\begin{tabular}{||c|c|c|c||}
\hline $\begin{array}{c}\text { DNA Conc } \\
(\mathrm{ng} / \mathrm{mL})\end{array}$ & $\begin{array}{c}\text { DNA Flow } \\
\text { Rate } \\
(\mu \mathrm{L} / \mathrm{s})\end{array}$ & $\begin{array}{c}\text { Wash Flow } \\
\text { Rate } \\
(\mu \mathrm{L} / \mathrm{s})\end{array}$ & $\begin{array}{c}\text { Capture } \\
\text { Efficiency } \\
(\%)\end{array}$ \\
\hline 1000 & 0.5 & 5.0 & $2.8^{1}$ \\
\hline 100 & 0.1 & 0.5 & 10 \\
\hline 100 & 0.5 & 0.5 & 10 \\
\hline 100 & 0.5 & 5.0 & 10 \\
\hline 100 & 5.0 & 5.0 & 5 \\
\hline \hline
\end{tabular}

${ }^{1}$ DNA capture is at the binding limit of the structure.

\section{LOW CONCENTRATION RESULTS}

For the low concentration studies, lambda DNA $(48,000$ bp) [10] was used as a bacteria simulant. Starting solutions were again prepared by dilution of lambda stock with chaotropic salt solutions. Starting copy number for the first set of experiments was $5 \times 10^{4}$ copies in $500 \mu \mathrm{L}$ of solution. As a control, the experimental protocol was first run using a chaotropic solution without DNA. Standards of known copy number $\left(10^{4}\right.$ and $\left.5 \times 10^{4}\right)$ were also prepared using both water that had passed through the chip test system, and pure water.

The protocol was similar to that of the high concentration studies. Typically, $500 \mu \mathrm{L}$ of the input test solution was passed through the chip at $0.5 \mu \mathrm{L} / \mathrm{s}$, followed by $250 \mu \mathrm{L}$ of wash solution. Following the wash, an air gap was introduced to delineate the wash/elution boundary. Elution agent was introduced into the chip, and allowed to stand for several minutes. Elution droplets were then collected directly into PCR reaction tubes. Four elution samples were taken from the chip, consisting of three samples of $25 \mu \mathrm{L}$ followed by an additional sample of $50 \mu \mathrm{L}$. The $25 \mu \mathrm{L}$ samples were diluted up to $50 \mu \mathrm{L}$ after collection so that all samples submitted to PCR were $50 \mu \mathrm{L}$. The $500 \mathrm{bp} \mathrm{PCR} \mathrm{products}$ were run through gel electrophoresis and photographs of the gel (under UV illuminuation) were taken and used to compare the chip elution samples to the standards of known copy number.

Fig. 5 shows the gel photograph from one such experiment. The standards prepared with system water are shown first, followed by the run with blank solution, the run with DNA solution, and standards prepared with pure water. By comparing the first $25 \mu \mathrm{L}$ chip elution to the $10^{4}$ and $5 \times 10^{4}$ standards in system water, it is estimated that the first chip elution contains about $2.5 \times 10^{4}$ copies. This implies a $10 \mathrm{X}$ concentration effect, and a $50 \%$ capture efficiency. There is a small amount of PCR inhibition evidenced by the fact that the $5 \times 10^{4}$ standard prepared using system water is not as intensely stained as the $5 \times 10^{4}$ standard prepared using pure water.

\section{CONCLUSIONS}

DNA analysis technology is playing an increasing role in many health-related areas. Laborious, manual procedures introduce human variability, possible cross contamination and high cost. By replacing such procedures with automated, disposable assays, the cost and applicability of this exciting new technology will gain the widest possible market. Automated bench-top and hand-held DNA analysis tools utilizing disposable cartridge formats are being developed to serve this need. As a component of such systems, the use of silicon microstructures for the extraction and concentration of target DNA can play an important role.

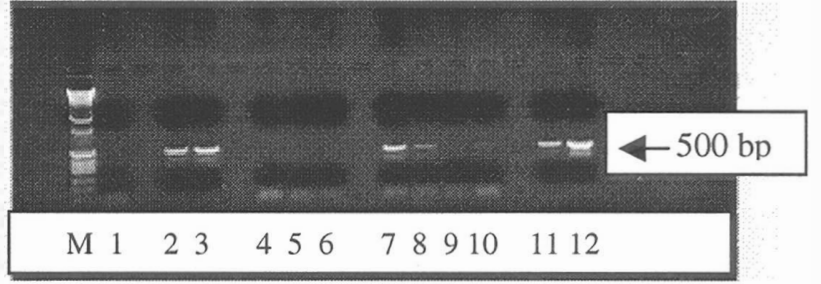

Figure 5. Photograph of electrophoretic gel showing DNA chip capture. Lanes: M: Molecular weight standard, 1: Neg Ctl, 2: $10^{4}$ standard in system water, 3: $5 \times 10^{4}$ standard in system water, 4-6: control run with no DNA, 7 - 10: Chip Elutions from DNA run, 11: $10^{4}$ standard in pure water, 12:5 $\times 10^{4}$ standard in pure water. By comparing lane 7, obtained from the first $25 \mu \mathrm{L}$ chip elution, to standard lanes 2 and 3, it is estimated that a capture efficiency of $50 \%$ was achieved, with a concentration factor of about $10 \mathrm{X}$ in the first elution.

We have shown that deep reactive ion etching can be used to produce high surface area structures suitable for use as DNA capture elements. With proper design of structure and protocol, we believe such devices will be critical, cost effective components in future DNA analysis systems.

\section{ACKNOWLEDGMENTS}

The authors would like to thank Rick Faeth for wafer fabrication, and Linda Western and Sandy Zaner for PCR processing. This work was supported in part by funds from the United States Army Medical Research Institute of Infectious Diseases (USAMRIID).

\section{REFERENCES}

1. J.K. Bhardwaj, H. Ashraf, "Advanced Silicon Etching using High Density Plasmas", SPIE Micromachining and Fabrication Technology Proc. 2639, pp. 224-233 (1995).

2. B. P. van Driëenhuizen, N. I. Maluf, I. E. Opris, and G. T. A. Kovacs, "Force-Balanced Accelerometer with mG Resolution, Fabricated using Silicon Fusion Bonding and Deep Reactive Ion Etching", Technical Digest of the 1997 International Conference on Solid-State Sensors and Actuators, Transducers '97, Chicago, IL, pp. 1229-1230 (1997).

3. R.K. Saiki, S. Scharf, F. Faloona, K.B. Mullis, G.T. Horn, H.A. Erlick and N. Arnheim, "Enzymatic amplification of $\beta$ globin genomic sequences and restriction site analysis for diagnosis of sickle cell anemia", Science 230, pp 1350-1354 (1985).

4. Biorad Labs, Hercules CA, Promega, Madison WI, and Qiagen Inc., Santa Clarita, CA.

5. Willem R. Boom et al, US Patent 5,234,809 (Aug 1993).

6. Christophe Marques, Yohannes M. Desta, James Rogers, Michael C. Murphy, and Kevin Kelly, "Fabrication of HighAspect-Ratio Microstructures on Planar and Nonplanar Surfaces Using a Modified LIGA Process", JMEMS 6(4), pp. 329-336 (1997).

7. Molecular Probes, Eugene OR. YOYO-1: P/N Y-3601, PicoGreen: P/N P7581.

8. Bio-Rad Low Range Fluorescein Standard P/N 170-3123.

9. Bert Vogelstein and David Gillespie, "Preparative and analytical purification of DNA from agarose", Proc. Natl. Acad. Sci., 76(2), pp. 615-619 (1979).

10. Pharmacia, Bridgewater, NJ. 